INRA Prod. Anim., 1997, 10 (1), 79-89
G. AUMONT, R. POUILLOT, R. SIMON, G. HOSTACHE, H. VARO, N. BARRE *

INRA Unité de Recherches Zootechniques BP 51597165 Pointe-à-Pitre Cedex, Guadeloupe (FWI)

* CIRAD-EMVT, BP 515, 97165 Pointe-à-Pitre Cedex, Guadeloupe (FWI)

\section{Parasitisme digestif des petits ruminants dans les Antilles françaises}

Depuis 15 ans, des informations sur le parasitisme digestif des petits ruminants ont été accumulées par l'INRA à la fois dans ses dispositifs expérimentaux et en ferme. Ces résultats concernent essentiellement les strongyloses gastro-intestinales, pathologie majeure des petits ruminants en milieu tropical humide. Ils touchent à la fois l'épidémiologie, les dynamiques des stades libres, la résistance aux anthelminthiques et la variabilité de la réceptivité des hôtes. La réussite technique de l'intensification, élément essentiel pour le maintien de l'élevage des petits ruminants comme activité économiquement viable dans la zone Caraïbe, passe par une maîtrise du parasitisme digestif. L'intérêt des données présentées dans cet article réside dans la diversité des milieux étudiés en Guadeloupe et en Martinique, milieux très fréquemment rencontrés dans l'ensemble de la zone Caraïbe. En outre, ces données concernent aussi les autres pays de la zone Caraïbe où les structures vétérinaires ne sont pas en mesure de fournir des informations de ce type.

\begin{abstract}
Résumé
Les parasitoses digestives, pathologie majeure des petits ruminants de la zone Caraïbe, sont dues essentiellement à une espèce de Nématode, Haemonchus contortus et, dans une moindre mesure, à une espèce de Cestode, Moniezia sp., et un ensemble de protozoaires du genre Eimeria. Des études en station expérimentale et en fermes ont permis de définir les dynamiques des larves infestantes d'Haemonchus contortus et de Trichostrongylus colubriformis sur le pâturage. Les risques d'infestation parasitaire ont été ensuite quantifiés par des modélisations et des simulations selon la saison, l'irrigation, la biomasse herbacée sur le pâturage et le mode de gestion du pâturage.

En Guadeloupe, la diversité faunistique des populations vermineuses est faible : 10 espèces. Les prévalences d'Haemonchus sp. et Trichostrongylus spp. varient entre 80 et $100 \%$. Les incidences dépassent très fréquemment $60 \%$ par mois pour ces genres. Les facteurs relatifs à l'eau (irrigation, régions sèches ou humides) ou à la réceptivité des hôtes (âge par exemple) sont les principales sources de variation du risque d'infestation parasitaire par les strongles digestifs. Plus des trois quarts de la mortalité avant le sevrage (soit $40 \%$ ) sont liés pour tout ou partie aux strongles gastro-intestinaux. L'utilisation fréquente des anthelminthiques a induit l'apparition de résistances d'Haemonchus contortus aux benzimidazoles dans toute la Guadeloupe et la Martinique. Les études en fermes ont confirmé que les strongyloses gastro-intestinales sont la pathologie dominante des petits ruminants des petites Antilles.

Les recherches pour améliorer les techniques d'élevage au pâturage menées à l'Unité de Recherches Zootechniques de l'INRA (rotation, pâturage mixte avec les bovins) tiennent compte des répercussions des strongyloses sur les productions. Des études sont menées sur la résistance génétique aux strongles digestifs chez les caprins Créoles et l'intérêt de complémentations alimentaires dans des plans de lutte intégrée contre ces parasitoses.
\end{abstract}

Dans toutes les zones intertropicales, les strongyloses gastro-intestinales représentent une pathologie majeure des petits ruminants domestiques, par leur fréquence et leurs conséquences zootechniques et économiques (Fabiyi 1987). En effet, leur prévalence est pratiquement égale à $100 \%$, même dans des milieux très secs comme les régions soudanosahéliennes (Vassiliades 1984), dès que des conditions d'humidité permettent l'achèvement de la partie externe de leur cycle. Cependant, leurs conséquences économiques sont sous-estimées. En effet, leur expression clinique est très souvent fruste. En outre, il n'existe pas d'approche simple qui permette de préciser les facteurs de variation des populations vermineuses ou larvaires, ainsi que leurs effets en termes de performances animales et économiques. Cependant, les pertes zootechniques globales engendrées par ces strongyloses gastro-intestinales (mortalité, croissance et reproduction) peuvent représenter jusqu'à $33 \%$ de la productivité des troupeaux au Sénégal (Faugère et al 1991), $11 \%$ au Tchad et au Nigeria (Schillhorn Van Veen 1973). Au Nigeria, l'importance de l'haemonchose pour l'économie de l'élevage des ovins et des caprins a été démontrée (Aberejola et al 
1979). La plus grande partie de ces pertes en milieu tropical est liée aux nématodes des genres Haemonchus, Trichostrongylus et Oesophagostomum (Fabiyi 1987).

Dans les Caraïbes, Williams (1990) estime que ces strongyloses gastro-intestinales représentent un groupe de pathologies majeures, dont l'importance zootechnique et économique est équivalente, voire supérieure, à celle des maladies associées aux tiques. Cependant, cette opinion reste fondée sur des observations cliniques fragmentées, des expériences de vétérinaires praticiens qui ne sont que rarement étayées par des diagnostics biologiques. Les structures d'abattage sont rarement conformes aux réglementations et l'abattage clandestin est très fréquemment pratiqué. Ceci ne permet pas d'obtenir des données indubitables que permettent les bilans parasitaires effectués à l'abattoir.

Les instituts de recherches dans les Départements Français d'Amérique (DFA) ont développé des modèles d'intensification au pâturage : irrigation, fortes charges, biomasse herbacée importante. Ces modèles se sont heurtés à une explosion massive des strongyloses gastro-intestinales et l'apparition de nombreuses résistances aux anthelminthiques. La lutte intégrée contre les strongyloses gastro-intestinales est apparue comme l'accompagnement essentiel de l'intensification fourragère dans la production des petits ruminants. En effet, dans de tels systèmes de production intensifs en élevage ovin (Martinique) ou caprin (Guadeloupe), la fréquence des traitements anthelminthiques doit dépasser dix traitements annuels pour le maintien des performances. Des travaux ont donc été engagés en Guadeloupe et, dans une moindre mesure, en Martinique, pour préciser l'importance de ces parasitoses : diversité faunistique, épidémiologie, écologie des stades libres, résistances aux anthelminthiques, résistance génétique des populations locales de petits ruminants. Les résultats de ces études font l'objet de cette synthèse.

Ces données présentent un intérêt pour les îles concernées mais aussi pour l'ensemble de la zone Caraïbe, archipel constitué d'une multitude de milieux agro-climatiques. Les types d'élevages y sont très divers. Mais l'archipel de la Guadeloupe rassemble des milieux très variés qui peuvent être retrouvés dans l'ensemble de la zone des petites Antilles (Lasserre 1961).

\section{$1 /$ Milieux et types d'élevage de petits ruminants de Guadeloupe et de Martinique}

\section{1 / La Guadeloupe}

La Guadeloupe est un archipel de huit îles habitées et d'une centaine d'îlots coralliens déserts, situé au milieu de l'arc volcanique des Petites Antilles $\left(16^{\circ} \mathrm{N}, 61^{\circ} \mathrm{O}\right)$. Elle est constituée de deux îles principales (la BasseTerre : $848 \mathrm{~km}^{2}$, et la Grande-Terre : $590 \mathrm{~km}^{2}$ ) sur lesquelles ont été accumulées les observations. La Basse-Terre dont le volcan (la Soufrière) culmine à $1467 \mathrm{~m}$, fait partie de l'arc interne fortement volcanisé des petites Antilles. Au nord, la Grande-Terre est une île essentiellement calcaire constituée de plaines argileuses et de plateaux calcaires et d'une région de « mornes " (collines) calcaires (les « Grands-Fonds »; Lasserre 1982).

Le climat est de type tropical humide. La température est élevée $\left(25,3{ }^{\circ} \mathrm{C}\right.$, amplitude annuelle $3,3^{\circ} \mathrm{C}$ ) avec une amplitude diurne de $8,8^{\circ} \mathrm{C}$. L'humidité relative est en moyenne d'environ $80 \%$, toujours supérieure à $50 \%$. La chaîne du massif de la Soufrière, perpendiculaire aux alizés, est assez élevée pour diviser la Basse-Terre en deux zones climatiques très différentes : un versant est ( côte au vent ») très arrosé, et un versant ouest (« côte sous le vent ») recevant peu de pluies. On peut donc distinguer 5 régions dans la Guadeloupe continentale selon la pluviométrie, le relief et les sols :

- le Massif de la Soufrière (MS), à plus de 5 $000 \mathrm{~mm} / \mathrm{an}$;

- la «Côte au vent » (CAV) de Basse-Terre avec une pluviométrie supérieure à 3000 $\mathrm{mm} / \mathrm{an}$;

- la "Côte sous le vent " de Basse-Terre (CSV), avec une pluviométrie variant entre 1 500 et $3000 \mathrm{~mm} / \mathrm{an}$;

- la région des "Grands-Fonds "(GF), région des collines de l'ouest de la GrandeTerre d'une pluviométrie variant de 1500 à 2 $500 \mathrm{~mm} / \mathrm{an}$;

- la région du Nord de la Grande-Terre (NGT), présentant une pluviométrie variant entre 1000 à $1500 \mathrm{~mm} / \mathrm{an}$.

Malgré une très grande variabilité interannuelle, la répartition des pluies permet de distinguer deux saisons : d'une part, la saison sèche ou "carême " entre janvier et avril, très marquée dans les régions Côte sous le vent, Grands Fonds et le Nord Grande-Terre et, d'autre part, la saison humide ou « hivernage ", à tendance équatoriale, avec de fortes pluies culminant en octobre.

Bien que le cheptel caprin en Guadeloupe soit important relativement aux autres cheptels de la zone Caraïbe (39 500 têtes ; INSEE 1993), l'élevage reste le plus souvent une activité annexe à l'exploitation agricole (Alexandre et al 1991). La production caprine connaît cependant une relative intensification. Le nombre moyen de caprins par exploitation était de 9,7 en 1989 et la moitié des exploitations ont moins de 4 animaux par exploitation (Tatareau et al 1991). La quasi totalité des caprins de Guadeloupe sont de race Créole. Cette population résulte de croisements d'animaux provenant des différents courants d'importation d'Afrique, d'Asie et d'Europe (Chemineau et al 1984). L'analyse du génome a confirmé la similitude de cette race avec la chèvre West African Dwarf (Pépin 1994). 
En station expérimentale, la race Créole présente de bonnes performances zootechniques (désaisonnement et grande prolificité : Chemineau et al 1984). La production à l'herbe dans des systèmes intensifs permet d'obtenir près de $25 \mathrm{~kg}$ de chevreaux sevrés par chèvre et par an (Alexandre et al 1997). Cependant, en élevage, les prairies sont le plus souvent des savanes ou des parcours conduisant à des performances beaucoup plus faibles. Quatre types de gestion des pâturages sont principalement rencontrés (Alexandre et Borel 1990) : élevage « au piquet » $(25 \%$ des élevages), pâturage continu (32\%), pâturage en rotation $(23 \%)$ et liberté $(20 \%)$.

Les pathologies principales sont liées aux parasites internes et aux parasites externes. Certains auteurs considèrent que la cowdriose et la dermatophilose, maladies liées à la présence de la tique Amblyomma variegatum, constituent l'essentiel des pertes en élevage bovin et caprin (Camus 1991, Petitclerc et al 1991). Cependant les résultats épidémiologiques qui fondent ces opinions restent limités.

\section{2 / La Martinique}

La Martinique est une île d'une superficie de $1090 \mathrm{~km}^{2}$ située au sud de la Guadeloupe. Sa structure et son climat sont similaires à la partie volcanique de la Guadeloupe. Trois régions peuvent être distinguées : au nord, des formations volcaniques (1 000-1 $500 \mathrm{~m}$ ) récentes à très forte pluviométrie (5 000 $\mathrm{mm} / \mathrm{an})$; au centre, une plaine barrée à l'est par une juxtaposition de mornes, et au sud une région de mornes à pluviométrie faible (1 000-1 $500 \mathrm{~mm} / \mathrm{an})$. C'est dans cette dernière région qu'est concentré l'élevage des petits ruminants, pratiquement exclusivement représenté par les ovins de race "Martinik ", mouton à poil de type Black Belly et d'origine Djallonké. Le cheptel de 43000 têtes est réparti dans des troupeaux de 10 têtes en moyenne (Tatareau et al 1991). En ferme, les niveaux de production sont faibles (Leimbacher et Tatareau 1991), mais l'intensification de la production à l'herbe (irrigation, fertilisation) à partir de cette population locale est possible (Mahieu 1991, Mahieu et al 1997). Cependant, la pathologie parasitaire devient alors un facteur très contraignant limitant le maintien de ce type de production.

\section{2 / Principes des recherches et aspects méthodologiques}

Les données d'inventaire faunistique obtenues dans les DFA sont issues de bilans parasitaires effectués le plus souvent par des enquêtes ponctuelles en Guadeloupe (Euzeby et Graber 1973, Lebigre 1979, Durand et al 1983, Esterre et Maitre 1985). Ces études sont entachées de biais liés à des échantillonnages généralement peu raisonnés. Ces don- nées ont été complétées par une enquête épidémiologique dont l'échantillonnage permet une estimation des effets des régions et des saisons (Simon 1994, Pouillot 1995).

Deux grands types de travaux ont été menés par l'INRA dans ces domaines. Des recherches d'amont en station expérimentale ont été effectuées jusqu'en 1985 pour préciser les facteurs de variation des dynamiques de population des stades infestants sur le pâturage. Ces données ont été confrontées aux réalités de terrain par une enquête épidémiologique sur les strongyloses gastro-intestinales caprines dans des fermes de Guadeloupe. Dans ce département, la taille des troupeaux est si petite et la diversité des situations d'élevage si grande qu'il n'était pas possible d'envisager des études épidémiologiques sur des bilans parasitaires. Une méthode originale a donc été élaborée pour obtenir des données épidémiologiques dynamiques et quantitatives dans un milieu insulaire particulier.

L'enquête épidémiologique a été fondée sur quatre passages successifs mensuels par saison (saison humide 1993 et saison sèche 1994) dans 21 exploitations choisies dans l'ensemble de la Guadeloupe continentale (Grande-Terre et Basse-Terre). Dans chacune des cinq régions, une bonne répartition des modes de gestion du pâturage et d'élevage (piquet, continu, rotation) a été obtenue. Pour chaque saison, un traitement per os à l'ivermectine a été administré au second passage dans les exploitations, afin d'obtenir des résultats d'incidence et des données non biaisées par des populations parasitaires résiduelles. A chaque passage, des données démographiques, cliniques, parasitologiques (nombre d'œufs de strongles dans les fèces, densités larvaires sur les prairies), biologiques chez l'hôte (hématocrite, albuminémie, taux de pepsinogène sérique), climatologiques (données quotidiennes de pluviométrie et de rayonnement global) ont été recueillies. Une approche quantitative des méthodes parasitaires les plus fréquemment utilisées a été développée. Les méthodes de coproscopie quantitative ou de détermination de la taille des populations larvaires sur le pâturage ont été améliorées et leur robustesse éprouvée (Aumont et al 1995a, 1996a), permettant ainsi d'apprécier les relations entre populations externes et populations internes.

\section{3 / Inventaire faunistique}

La liste des espèces rencontrées en Guadeloupe (tableau 1) montre une diversité faunistique limitée : 15 espèces de nématodes, 4 espèces de cestodes et 1 espèce de trématode. Haemonchus contortus, Trichostrongylus colubriformis et Oesophagostomum columbianum sont les principales espèces de strongles digestifs. Ce parasitisme est associé à des infections par Eimeria chez les ovins (E. parva, E. ovnoidalis, E. faurei, E. intricata) et les caprins (E. alijevi, $E$. ninakohlya- 
Tableau 1. Inventaire faunistique des helminthes parasites de caprins en Guadeloupe (FWI). Références : (1) Euzéby et Graber 1973, (2) Lebigre 1979, (3) Peroux 1982, (4) Esterre et Maître 1985, (5) Durand et al 1983, (6) Simon 1994, (7) Pouillot 1995.

\begin{tabular}{|c|c|c|c|c|c|c|c|}
\hline Référence & (1) & (2) & (3) & (4) & (5) & (6) & (7) \\
\hline $\begin{array}{l}\text { Larynx } \\
\text { Mammomonogamus nasicola }\end{array}$ & - & $\mathrm{X}$ & - & - & - & $\mathrm{X}$ & $\mathrm{X}$ \\
\hline $\begin{array}{l}\text { Poumons } \\
\text { Protostrongylus rufescens }\end{array}$ & - & $\mathrm{X}$ & - & - & & $X$ & \\
\hline $\begin{array}{l}\text { Rumen } \\
\text { Cotylophoron cotylophorum }\end{array}$ & - & $\mathrm{X}$ & - & $\mathrm{X}$ & - & $\mathrm{X}$ & $\mathrm{X}$ \\
\hline Abomasum & & & & & & & \\
\hline $\begin{array}{l}\text { Haemonchus contortus } \\
\text { Trichostrongvlus axei }\end{array}$ & - & $\mathrm{X}$ & $\mathrm{X}$ & $\mathrm{X}$ & $\mathrm{X}$ & $\mathrm{X}$ & $\mathrm{X}$ \\
\hline $\begin{array}{l}\text { Trichostrongylus axei } \\
\text { Mecistocirrus digitatus }\end{array}$ & $\bar{x}$ & $\mathrm{X}$ & $\mathrm{X}$ & $\begin{array}{c}X \\
-\end{array}$ & - & $\begin{array}{l}X \\
-\end{array}$ & $\begin{array}{c}X \\
-\end{array}$ \\
\hline Teladorsagia circumcincta & - & $\mathrm{X}$ & - & $\mathrm{X}$ & $\mathrm{X}$ & - & - \\
\hline Intestin grêle & & & & & & & \\
\hline Strongyloides papillosus & - & $\mathrm{X}$ & - & - & - & $\mathrm{X}$ & $\mathrm{X}$ \\
\hline Trichostrongylus colubriformis & - & & $\mathrm{X}$ & $\mathrm{X}$ & $\mathrm{X}$ & $\mathrm{X}$ & $\mathrm{X}$ \\
\hline Cooperia spp. & - & $\mathrm{X}$ & & - & & - & 1 \\
\hline Bunostomum trigonocephalum & - & $\mathrm{X}$ & - & - & - & - & - \\
\hline Moniezia benedeni & - & $\mathrm{X}$ & - & - & - & - & - \\
\hline Moniezia expansa & $\mathrm{X}$ & $\mathrm{X}$ & $\mathrm{X}$ & $\mathrm{X}$ & $\mathrm{X}$ & $\mathrm{X}$ & $\mathrm{X}$ \\
\hline Thysaniezia giardi & - & $\mathrm{X}$ & - & $\mathrm{X}$ & - & - & - \\
\hline Avitellina centripunctata & - & $\mathrm{X}$ & - & - & - & - & - \\
\hline Gros intestin & & & & & & & \\
\hline Trichuris ovis & - & $\mathrm{X}$ & - & - & $\mathrm{X}$ & - & $\mathrm{X}$ \\
\hline Trichuris globulosa & 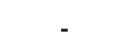 & & & 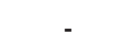 & & $\mathrm{X}$ & \\
\hline Oesophagostomum columbianum & $\mathrm{X}$ & $\mathrm{X}$ & - & $\mathrm{X}$ & $\mathrm{X}$ & $\mathrm{X}$ & $\mathrm{X}$ \\
\hline Skrjabinema ovis & 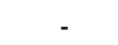 & $\mathrm{X}$ & - & - & - & - & - \\
\hline Nombre de bilans & 4 & 50 & 47 & $14+47$ & 15 & 19 & 15 \\
\hline Nombre de coproscopies & - & & & - & 41 & 1039 & 962 \\
\hline Provenance & abattoir & abattoir & INRA & $\begin{array}{l}\text { abattoir } \\
\text { et INRA }\end{array}$ & 5 élev. & 20 élev. & 20 élev \\
\hline
\end{tabular}

Chez les caprins en Guadeloupe et les ovins en

Martinique, le nombre d'espèces de strongles rencontrées est limité à une dizaine, avec 3 espèces principales dont Haemonchus contortus. kimovae, E. arloingi, E. christenseni). Ces résultats divergent légèrement des quelques données de la littérature établies sur l'ensemble des autres îles de la Caraïbe (Singh 1993). Cependant cet auteur fait état de travaux peu convaincants car la diversité faunistique observée dans chaque île est souvent très limitée.

Les espèces de strongles digestifs rencontrées chez les ovins de Martinique sont similaires à celles des caprins en Guadeloupe. Comme chez les caprins, la prévalence d'Haemonchus contortus est égale à $100 \%$. Les fréquences des phénotypes des vers femelles « bouton ", "lisse " et "languette " (Das et Whitlock 1960) suggèrent que c'est l'écotype Haemonchus contortus utkalensis qui est présent en Guadeloupe (Peroux 1982, N. Barré, résultats non publiés) et en Martinique (Gruner et al 1986, Bastien et al 1991). Il faut noter que T. axei n'a pas été observé chez les ovins. L'association avec des bovins au pâturage induit l'apparition d'infestations légères par Cooperia punctata et Cooperia pectinata chez les ovins (Aumont et al 1995b).

Ces études, basées sur un nombre limité de bilans, ne permettent pas en principe d'obte- nir une estimation de l'importance respective des différentes populations vermineuses ainsi que de leurs principales sources de variation. Toutefois, on peut admettre que l'intensification au pâturage induit une augmentation de la prévalence de Moniezia spp. En effet celleci varie entre 6 et $21 \%$ en ferme chez les caprins alors qu'en station expérimentale les prévalences varient entre $35 \%$ et $100 \%$ chez les jeunes avant 5 mois, malgré des traitement à base de niclosamide (Gruner et al 1984a, Mandonnet 1995). Ces systèmes intensifs stéréotypés diminuent la diversité des espèces puisque seules 3 espèces de strongles (Haemonchus contortus, Trichostrongylus colubriformis et Oesophagostomum columbianum) et une espèce de cestode (M. expansa) sont observées dans les domaines expérimentaux chez les ovins et les caprins.

La saison ne modifie que très légèrement les prévalences des différentes espèces de strongles calculées sur des bilans parasitaires de caprins en ferme, la différence la plus notable étant une augmentation de la prévalence d'Oesophagostomum columbianum en saison humide (tableau 2). 


\section{4 / Facteurs de variation des populations larvaires et vermineuses des strongles gastro-intestinaux}

\section{1 / Dynamique des stades infestants d'Haemonchus sp. et de Trichostrongylus spp. sur le pâturage}

En Guadeloupe, dans la région Nord Grande-Terre, des larves infestantes pour les petits ruminants du stade 3 (L3) des genres Haemonchus ou Trichostrongylus peuvent être détectées dès le $8^{\mathrm{e}}$ jour après le début d'un dépôt de matière fécale. Après un dépôt d'une durée de 7 jours, le maximum de L3 sur le pâturage est observé entre le $14^{\mathrm{e}}$ et le $21^{\mathrm{e}}$ jour après un dépôt de fèces. La durée de survie varie entre 47 et 56 jours en saison humide (Aumont et Gruner 1989). La taille de la population larvaire sur l'herbe varie d'un facteur 5 selon l'heure du nycthémère, mais il n'est pas possible de définir une loi simple de migration verticale de larves sur le couvert végétal, telle qu'elle a pu être envisagée pour des régions tempérées par Messean (1981). Durant la saison sèche, le taux de développement des œufs en L3 est 5 fois plus faible que durant la saison humide (Aumont et Gruner 1989). L'eau (irrigation) et une biomasse fourragère importante sont des facteurs favorisant le taux de développement des œufs en larves (Gruner et al 1989, Berbigier et al 1990). Des rayonnements solaires importants diminuent le taux de développement des œufs en L3 (Gruner et al 1989, Berbigier et al 1990). La saison est alors un élément déterminant de variation des dynamiques larvaires sur le pâturage en milieu non irrigué. Ces résultats sont en accord avec ce qui est connu dans d'autres climats tropicaux (Ikeme et al 1986, Banks et al 1990, Krecek et al 1991). Les œufs embryonnés et les larves du genre Trichostrongylus présentent une résistance à la sécheresse supérieure à ceux du genre Haemonchus (Berbigier et al 1990) comme cela a déjà été noté par Waller et Donald (1970), Levine et Todd (1975), Levine et al (1977) et Wharton (1982).

Les données ont été ajustées à l'aide de modèles de croissance et de mortalité développés pour les populations de larves infestantes d'Haemonchus placei et Cooperia spp en Guadeloupe (Aumont et al 1989). Ces modèles ont servi à des simulations sur ordinateur pour construire des abaques de risque d'infestation, intégrant la charge, le mode de gestion du pâturage (durée de séjour, nombre de parcelles et âge repousse), la masse fourragère et l'irrigation (Aumont et al 1991). Ces simulations montrent que le passage d'un système sec (charge animale de $800 \mathrm{~kg} / \mathrm{ha}$ et 35 jours d'âge repousse) à un système intensif (charge animale de $1200 \mathrm{~kg} / \mathrm{ha}$, irrigation, fertilisation importante provoquant une forte biomasse fourragère et 28 jours d'âge repousse) multiplie par 16 le risque d'infestation parasitaire.
Tableau 2. Fréquence de l'espèce dans la communauté des vers et prévalence (fréquence du nombre d'animaux présentant l'espèce) chez des caprins de Guadeloupe continentale (Basse-Terre et Grande-Terre). Bilans effectués sur des animaux de moins d'un an et issus d'exploitations privées (Saison sèche : Simon 1994, saison humide : Pouillot 1995).

\begin{tabular}{|l|c|c|c|c|}
\hline & \multicolumn{2}{|c|}{ Saison sèche } & \multicolumn{2}{c|}{ Saison humide } \\
\cline { 2 - 5 } & $\begin{array}{c}\text { Fréquence } \\
\%\end{array}$ & $\begin{array}{c}\text { Prévalence } \\
\%\end{array}$ & $\begin{array}{c}\text { Fréquence } \\
\%\end{array}$ & $\begin{array}{c}\text { Prévalence } \\
\%\end{array}$ \\
\hline Mammomonogamus nasicola & 0,02 & 15,79 & 0,01 & 20,00 \\
Protostrongylus rufescens & 0,02 & 5,26 & 0,00 & 0,00 \\
Cotylophoron cotylophorum & 0,44 & 36,84 & 0,26 & 26,67 \\
Haemonchus contortus & 44,42 & 100,00 & 23,71 & 100,00 \\
Trichostrongylus axei & 2,44 & 52,63 & 11,82 & 46,67 \\
Trichostrongylus colubriformis & 48,90 & 84,21 & 58,27 & 93,33 \\
Strongyloides papillosus & 3,37 & 26,32 & 4,92 & 53,33 \\
Moniezia expansa & 0,07 & 26,32 & 0,00 & 6,67 \\
Trichuris ovis & 0,10 & 21,05 & 0,22 & 20,00 \\
Oesophagostomum columbianum & 0,33 & 26,32 & 0,68 & 80,00 \\
Nombre de bilans & & 19 & & 15 \\
\hline
\end{tabular}

\section{2 / Dynamique des stades infestants de strongles gastro-intestinaux des caprins en Guadeloupe : données en ferme}

Les méthodes d'estimation quantitative des densités larvaires sur le pâturage, des concentrations d'œufs de strongles dans les fèces (OPG), l'estimation des charges animales et l'application des modèles de dynamique des populations ont permis la détermination d'indice de développement des œufs en L3 en ferme. Ceci a permis une interprétation des variations de taille des populations larvaires en tenant compte du nombre d'œufs de strongles déposés par les animaux (Simon et al 1996).

En Guadeloupe, les densités larvaires observées sur les parcelles des fermes sont considérables, les médianes étant de 2150 et $3397 \mathrm{~L} 3 / \mathrm{kg}$ de matière sèche (MS) d'herbe respectivement en saison sèche et saison humide. Au cours de l'enquête, malgré une saison sèche très intense, la fréquence des parcelles présentant une population détectable de L3 (Haemonchus et Trichostrongylus) a été supérieure à $86 \%$, suggérant que le cycle de ces strongles peut être reproduit tout au long de l'année. Le rayonnement solaire global est le premier critère climatique qui semble intervenir sur les variations de la densité des populations larvaires d'Haemonchus et Trichostrongylus (Simon et al 1996). A l'inverse, les densités larvaires d'Oesophagostomum sont corrélées à la pluviométrie. En saison humide, il n'est pas possible de mettre en évidence de relation entre des variables du climat et les tailles des populations larvaires. Les proportions du genre Trichostrongylus dans les populations de L3 sont plus importantes dans les régions sèches (NGT, GF, CSV) que dans les régions humides (Pouillot 1995, Simon et al 1996 ; tableau 3). Ces obser-
L'eau d'irrigation et une biomasse fourragère importante favorisent le développement des oeufs en larves. 
Tableau 3. Densités larvaires (moyenne géométrique, en L3/kg MS) toutes espèces confondues, d'H. contortus ( $\mathrm{L} 3 \mathrm{Hae}$ ) et de T. colubriformis (L3 Tri) observées dans différentes régions de Guadeloupe en 1993 et 1994 (description des régions dans le texte). Saison sèche : Simon 1994, saison humide : Pouillot 1995.

\begin{tabular}{|l|r|r|r|r|r|r|}
\hline \multirow{2}{*}{ Région } & \multicolumn{3}{|c|}{ Saison sèche } & \multicolumn{3}{c|}{ Saison humide } \\
\cline { 2 - 7 } & Toutes L3 & L3 Hae & L3 Tri & Toutes L3 & L3 Hae & L3 Tri \\
\hline Grands-Fonds & $566^{\mathrm{a}}$ & $263^{\mathrm{a}}$ & $372^{\mathrm{a}}$ & $2122^{\mathrm{a}}$ & $409^{\mathrm{a}}$ & $1080^{\mathrm{a}}$ \\
Nord Grande-Terre ni $^{(1)}$ & $624^{\mathrm{a}}$ & $154^{\mathrm{a}}$ & $895^{\mathrm{a}}$ & $2893^{\mathrm{a}}$ & $1059^{\mathrm{a}}$ & $1503^{\mathrm{a}}$ \\
${\text { Nord Grande-Terre } \mathrm{i}^{(1)}}_{\text {Côte au Vent }}^{4537^{\mathrm{b}}}$ & $1092^{\mathrm{ab}}$ & $2057^{\mathrm{b}}$ & & & \\
Côte sous le Vent & $6195^{\mathrm{b}}$ & $3217^{\mathrm{a}}$ & $377^{\mathrm{a}}$ & $6438^{\mathrm{b}}$ & $3077^{\mathrm{b}}$ & $1874^{\mathrm{a}}$ \\
Massif de la Soufrière & $2040^{\mathrm{a}}$ & $959^{\mathrm{ab}}$ & $457^{\mathrm{a}}$ & $2752^{\mathrm{a}}$ & $1417^{\mathrm{ab}}$ & $9^{\mathrm{a}}$ \\
\hline
\end{tabular}

Dans une même colonne, les moyennes suivies de lettres différentes sont significativement différentes au seuil de $1 \%$.

(1) ni : parcelles non irriguées, i : parcelles irriguées.

vations sont similaires à celles effectuées à Ste Croix (Virgin Islands), îles sèches du nord de la zone Caraïbe (Courtney et Wildeus 1991). L'irrigation induit une augmentation d'un facteur 7 des populations larvaires et des indices de développement des œufs en L3 au cours de la saison sèche principalement (Gruner et al 1989).

L'ensemble de ces résultats montre une bonne adéquation entre les réalités de terrain et les données obtenues en station expérimentale. Cependant, les parcs et les élevages restent les principales sources de variation de la densité larvaire sur le pâturage (Simon 1994, Pouillot 1995). Des corrélations positives ont été notées entre les densités larvaires et les quantités d'œufs déposées durant les enquêtes épidémiologiques. Des régions et des saisons à risque ont pu être mises en évidence. Cependant, il reste à préciser s'il existe une relation entre ces populations larvaires très importantes sur les pâturages, y compris en saison sèche, et le niveau et la nature des infestations par les strongles gastro-intestinaux chez les petits ruminants.

\section{3 / Facteurs de variation des populations vermineuses chez les petits ruminants}

En station expérimentale, les systèmes basés sur de fortes charges instantanées et une rotation inférieure ou égale à 28 jours

Tableau 4. Concentration des œufs de strongles dans les fèces (œufs/g de fèces, genre Haemonchus essentiellement) de caprins (Guadeloupe : Mandonnet et al 1996) et d'ovins (Martinique : Aumont et al 1996) élevés sur pâturage irrigué de pangola (Digitaria decumbens) en système de rotation (28 jours d'âge repousse) et une charge globale de 1 à 1,2 t/ha. Moyenne géométrique 7 à 8 semaines après un traitement per os non rémanent (caprins : ivermectine, ovins : lévamisole).

\begin{tabular}{|l|c|c|c|c|}
\hline \multirow{2}{*}{} & \multicolumn{2}{|c|}{ Caprins (7-10 mois) } & \multicolumn{2}{c|}{ Ovins (6 mois) } \\
\cline { 2 - 5 } & Mâles & Femelles & Mâles & Femelles \\
\hline Saison humide (novembre-décembre) & 868 & 489 & 1000 & 358 \\
Saison sèche (janvier-mars) & 528 & 645 & 171 & 3 \\
Saison intermédiaire (juillet-août) & 1321 & 1052 & 668 & 175 \\
\hline
\end{tabular}

d'âge repousse induisent une explosion de l'infestation des caprins et des ovins par les strongles gastro-intestinaux, obligeant à des traitements mensuels pour les jeunes avant le sevrage. L'infestation massive, essentiellement par Haemonchus contortus, a lieu dès les premiers jours de la vie chez les caprins (Peroux 1982, Gruner et al 1984b). Dans ces systèmes, chez les caprins et les ovins, malgré l'irrigation, les niveaux d'infestation essentiellement par Haemonchus contortus en saison sèche sont inférieurs à ceux de la saison humide ou intermédiaire (tableau 4). Dans les deux espèces, les mâles sont plus infestés que les femelles (caprins : Mandonnet et al 1996, ovins : Aumont et al 1995c). Cependant ces indications restent limitées à un site et à un type de gestion du pâturage, système très peu répandu dans l'ensemble de la zone Caraïbe. A l'inverse, les données obtenues en ferme ne mettent pas en évidence d'effets saisonniers très nets sur la nature et la taille des populations vermineuses, ni même sur les populations larvaires sur les pâturages (Aumont et al 1996b).

Les données obtenues au cours d'enquêtes épidémiologiques en ferme permettent d'estimer des prévalences et des incidences sur un nombre important d'observations fécales (tableau 5) qui complètent les données des bilans parasitaires. La mortalité observée dans les élevages de caprins en Guadeloupe est proche de $45 \%$ pour les jeunes avant le sevrage. En se fondant sur des critères cliniques, parasitaires et biologiques, la mortalité annuelle estimée à partir des observations saisonnières et liée aux strongyloses gastrointestinales est considérable puisque près de $45 \%$ des jeunes avant le sevrage et $20 \%$ des jeunes élèves sont victimes de ces parasitoses (tableau 6). Les prévalences des principaux strongles gastro-intestinaux, et en particulier d'Haemonchus, proches de $80 \%$ expliquent largement ces mortalités. Les incidences de ces parasitoses sont extrêmement élevées dans les régions humides comme dans les régions sèches, en saison humide comme en saison sèche, en accord avec les très fortes densités larvaires observées sur les pâturages. En outre, en saison sèche, la fréquence 
Tableau 5. Incidence (\%/mois) des principaux parasites du tube digestif des caprins en Guadeloupe en saison sèche (Simon 1994) et en saison humide (Pouillot 1995). Les incidences sont calculées comme étant la fréquence des cas positifs après coproculture sur l'ensemble des échantillons fécaux collectés après un traitement à l'ivermectine per os (délai de 4 semaines pour Haemonchus contortus $(H c)$ + Trichostrongylus axei ou colubriformis (Tspp) et de 8 semaines pour Oesophagostomum columbianum (Oc)).

\begin{tabular}{|c|c|c|c|c|c|c|c|c|}
\hline & \multicolumn{4}{|c|}{ Saison sèche } & \multicolumn{4}{|c|}{ Saison humide } \\
\hline & Effectif & $\begin{array}{l}\mathrm{Hc} \\
\% / \text { mois }\end{array}$ & $\begin{array}{l}\text { Tspp } \\
\% / \text { mois }\end{array}$ & $\begin{array}{c}\text { Oc } \\
\% / 2 \text { mois }\end{array}$ & Effectif & $\begin{array}{c}\mathrm{Hc} \\
\% / \text { mois }\end{array}$ & $\begin{array}{l}\text { Tspp } \\
\% / \text { mois }\end{array}$ & $\begin{array}{c}\text { Oc } \\
\% / 2 \text { mois }\end{array}$ \\
\hline $\begin{array}{l}\text { Global } \\
\text { Age }\end{array}$ & 330 & 65,2 & 58,8 & $\begin{array}{l}47,0 \\
* * *\end{array}$ & 205 & $\begin{array}{c}54,1 \\
* *\end{array}$ & $\begin{array}{l}49,3 \\
* * *\end{array}$ & 28,7 \\
\hline $\begin{array}{l}\text { Age } \\
\text { moins de } 3 \text { mois }\end{array}$ & 44 & $\begin{array}{c}\mathrm{ns} \\
63,6\end{array}$ & $\begin{array}{c}\mathrm{ns} \\
50,0\end{array}$ & $\begin{array}{r}* * * \\
5,6\end{array}$ & 9 & $\begin{array}{l}* * \\
66,7\end{array}$ & 66,7 & $\begin{array}{c}\text { ns } \\
-\end{array}$ \\
\hline 3 à 18 mois & 133 & 52,6 & 45,9 & 45,1 & 76 & 74,5 & 72,6 & 28,3 \\
\hline plus de 18 mois & 153 & 62,8 & 47,1 & 32,9 & 120 & 42,6 & 36,2 & 28,9 \\
\hline Région & & ns & $* * *$ & $* * *$ & & $* * *$ & $* * *$ & ns \\
\hline Grands-Fonds & 83 & 51,8 & 41,0 & 57,0 & 52 & 52,7 & 52,8 & 31,0 \\
\hline Nord Grande-Terre & 100 & 57,0 & 58,0 & 23,9 & 63 & 51,2 & 65,2 & 53,3 \\
\hline Côte au Vent & 67 & 70,2 & 47,8 & 17,5 & 35 & 68,7 & 34,4 & 10,0 \\
\hline Côte sous le Vent & 46 & 47,8 & 23,9 & 43,2 & 42 & 25,0 & 16,7 & 10,0 \\
\hline Massif Soufrière & 34 & 75,5 & 58,8 & 55,2 & 13 & 84,6 & 84,6 & 15,0 \\
\hline Mode de pâturage & & ns & ns & $* *$ & $\mathrm{~ns}$ & & $\mathrm{~ns}$ & ns \\
\hline Piquet & 84 & 61,9 & 50,0 & 55,1 & 51 & 65,5 & 79,3 & 21,7 \\
\hline Rotation & 124 & 57,3 & 50,8 & 31,6 & 76 & 61,4 & 48,6 & 39,7 \\
\hline Continu & 122 & 58,2 & 41,0 & 27,4 & 76 & 36,7 & 32,7 & 18,6 \\
\hline
\end{tabular}

Test du Chi2 : ** $\mathrm{P}<0,01 ; * * * \mathrm{P}<0,001 ;$ ns : non significatif.

du genre Trichostrongylus dans les larves issues de coprocultures sont plus importantes dans les régions sèches de la Grande-Terre que dans les régions humides de Basse-Terre (Simon 1994), confirmant ainsi les observations de Courtney et Wildeus (1991) dans les îles Vierges.

La fréquence des animaux présentant une coproscopie négative aux strongles gastrointestinaux $(14,4 \%$ en saison sèche et $16,8 \%$ en saison humide) n'est pas associée à des indicateurs biologiques et cliniques de bonne santé (hématocrite, albuminémie, taux de pepsinogène sérique et indices cliniques). On peut observer fréquemment des animaux en très mauvais état clinique, indicateur de parasitoses sévères, alors qu'ils présentent des concentrations nulles d'œufs de strongles dans les fèces (OPG) et qu'ils pâturent des prairies très contaminées par des larves infestantes. La distribution particulière des variables de coproscopie (forte contribution des valeurs nulles), les très fortes densités larvaires sur le terrain et le mauvais état clinique de ces animaux suggèrent une fréquence importante de phénomènes d'autolibération ("self-cure »). Toutefois, il n'existe pas de critère indicateur simple de réaction immunitaire d'hypersensibilité de type 1 pour confirmer cet élément déterminant de l'épidémiologie de ces parasitoses dans la zone Caraïbe. Cependant, des cas avérés et bien décrits de "self-cure " massifs chez des ovins et des caprins ont été rapportés en station expérimentale (Mandonnet 1995, Mahieu et al 1996,).

Les principaux facteurs de variation de l'OPG et des critères biologiques chez les caprins en Guadeloupe (hématocrite, taux de pepsinogène sérique et albuminémie) sont la saison, la région, l'élevage et l'âge des animaux (tableau 7). Cependant, il subsiste une part importante de variation de ces paramètres non expliquée par ces facteurs, probablement liée à "l'histoire " parasitaire, immunitaire (phénomène de "self-cure ») et au potentiel génétique de résistance de chaque animal. L'estimation de ces facteurs n'est pas possible en ferme. Les animaux présentent des OPG élevés et un tableau clinique et biologique souvent très sombre, en accord avec les mortalités observées et les densités larvaires relevées sur les parcelles. Il convient de noter qu'il n'existe pas d'élément permettant d'estimer les interactions entre ces paramètres et la nature et la qualité de l'alimentation que reçoivent ces animaux. Cependant, les notes d'état corporel relevées chez les caprins en Guadeloupe sont significativement corrélées avec les OPG et les hématocrites (Simon 1994). Malgré le déficit fourrager, la saison sèche est une période qui semble mieux convenir aux caprins que la saison humide. La côte sous le vent, zone sèche où les ani-

\section{Dans les élevages caprins de Guadeloupe, la mortalité des jeunes avant sevrage atteint près de $45 \%$ par an dont $80 \%$ sont dus aux strongyloses gastro-intestinales.}

Tableau 6. Mortalités observées chez les caprins en ferme en Guadeloupe (1994-1995) et mortalité annuelle liée aux strongles gastro-intestinaux (SGI) selon des critères post-mortem (bilans parasitaires) et d'indicateurs parasitaires et biologiques (OPG, albuminémie, hématocrite). Saison sèche : 330 animaux contrôlés (Simon 1994), saison humide : 260 animaux contrôlés (Pouillot 1995).

\begin{tabular}{|l|c|c|c|}
\hline Age des animaux & $\begin{array}{c}\text { Saison sèche } \\
\% / 4 \text { mois }\end{array}$ & $\begin{array}{c}\text { Saison humide } \\
\% / 4 \text { mois }\end{array}$ & $\begin{array}{c}\text { Mortalité liée aux SGI } \\
\% / \text { an }\end{array}$ \\
\hline moins de 3 mois & 13,7 & 17,1 & 44,8 \\
3 à 18 mois & 8,2 & 15,9 & 20,7 \\
plus de 18 mois & 2,4 & 2,9 & 5,1 \\
\hline
\end{tabular}


Tableau 7. Concentration des œufs de strongles dans les fèces (OPG, moyenne géométrique estimée), hématocrite (héma), albuminémie (Alb) et note d'état clinique (NEC) chez des caprins de Guadeloupe selon la saison, l'âge, et la région (saison sèche : Simon 1994, saison humide : Pouillot 1995).

\begin{tabular}{|l|c|c|c|c|c|c|c|c|}
\hline & \multicolumn{4}{|c|}{ Saison sèche } & \multicolumn{3}{c|}{ Saison humide } \\
\cline { 2 - 9 } & $\begin{array}{c}\text { OPG } \\
\text { œufs/g }\end{array}$ & $\begin{array}{c}\text { héma } \\
\%\end{array}$ & $\begin{array}{c}\text { Alb } \\
\text { g/l }\end{array}$ & NEC & $\begin{array}{c}\text { OPG } \\
\text { œufs/g }\end{array}$ & $\begin{array}{c}\text { héma } \\
\%\end{array}$ & $\begin{array}{c}\text { Alb } \\
\text { g/l }\end{array}$ & NEC \\
\hline Age & & & & & & & & \\
moins de 3 mois & 1221 & 23,5 & 37,5 & 4,45 & 776 & 25,6 & 46,6 & 3,48 \\
3 à 18 mois & 494 & 27,8 & 40,4 & 4,86 & 751 & 27,1 & 46,7 & 3,43 \\
plus de 18 mois & 379 & 27,4 & 39,9 & 4,95 & 557 & 26,3 & 46,1 & 3,45 \\
Région & & & & & & & & \\
Grands-Fonds & 446 & 26,8 & 40,5 & 4,64 & 500 & 26,8 & 46,9 & 3,31 \\
Nord Grande-Terre & 741 & 25,4 & 38,4 & 4,29 & 490 & 27,3 & 47,8 & 3,09 \\
Côte au Vent & 921 & 25,6 & 37,3 & 4,86 & 1412 & 23,1 & 42,3 & 4,25 \\
Côte sous le Vent & 592 & 28,7 & 40,4 & 5,33 & 386 & 30,5 & 46,4 & 3,86 \\
Massif Soufrière & 476 & 24,7 & 39,7 & 4,64 & 1148 & 25,0 & 48,0 & 3,74 \\
\hline
\end{tabular}

NEC : Note d'état clinique variant de 1 à 10 estimée par des corrélations canoniques entre d'une part les OPG, PCV, Alb, Peps et d'autre part les notes d'état corporel, des phanères, d'estimation du ballonnement et d'estimation de la couleur des muqueuses oculaires (G. Aumont et al, résultats non publiés).

maux sont souvent élevés de façon extensive sur de grandes surfaces de parcours boisés, et la région des Grands-Fonds, où l'élevage se pratique souvent au piquet de façon traditionnelle, apparaissent comme les régions les plus favorables à l'élevage des petits ruminants. Dans aucune des études menées jusqu'alors, tant en ferme qu'en station expérimentale, il n'a été possible de mettre en évidence de relations claires entre taille des populations larvaires sur les pâturages et populations vermineuses chez les animaux au niveau de l'élevage. Les effets saisonniers sur la nature et la taille des populations parasitaires chez les caprins sont limités. En outre, dans la mesure où dans les fermes les modes de gestion du pâturage sont souvent très variables pour une même exploitation et rarement suivis avec rigueur, ils n'apparaissent pas comme des facteurs importants du niveau d'infestation parasitaire des animaux.

gestion très

rigoureuse du pâturage.

\section{5 / Epidémiologie des strongyloses gastro-intestinales des petits ruminants dans les Antilles françaises}

Les enquêtes en ferme selon une méthodologie appropriée à la faible taille des exploitations et à la diversité des milieux insulaires antillais, ainsi que les informations de base apportées par les études en stations expérimentales ont permis d'apporter des éléments de compréhension sur l'incidence clinique et zootechnique des strongyloses gastro-intestinales chez les caprins. De nombreuses informations peuvent en être déduites pour l'élevage des ovins en Martinique.

Les strongyloses gastro-intestinales sont très fréquentes chez les caprins en Guade- loupe. L'infestation par ces parasites est la règle générale dans tous les troupeaux étudiés. Haemonchus contortus et, dans une moindre mesure, Trichostrongylus spp. en particulier dans les zones et/ou en saison sèche, sont les principaux responsables de la pathologie endo-parasitaire. Les populations larvaires sur les prairies sont très importantes, même dans les situations et au cours des périodes les plus sèches de la Guadeloupe continentale (les deux principales îles, Grande-Terre et Basse-Terre). Cela se traduit par des niveaux élevés d'infestation des animaux par les genres Haemonchus et Trichostrongylus, des mortalités très fortes qui peuvent décimer des élevages (absence de jeunes de moins de 3 mois dans certaines fermes malgré de bons taux de fertilité) et des tableaux cliniques très sombres. Les phénomènes d'auto-libération sont probablement très fréquents. Le type de région est un des facteurs de variation importants de l'intensité et de la sévérité des strongyloses gastro-intestinales, mais une part importante de la variabilité animale observée est liée à l'élevage et à l'animal.

Ainsi, même si l'intensification au pâturage est certainement un mode d'élevage induisant une très forte augmentation du risque d'infestation par les strongles gastro-intestinaux, les élevages caprins de type plus traditionnel sont eux aussi soumis à une contrainte parasitaire forte.

\section{Conclusions : les moyens de lutte et l'orientation des recherches}

Des recommandations pratiques pour limiter l'infestation parasitaire ont pu ainsi être diffusées auprès des éleveurs de Guadeloupe et de Martinique à partir des informations 
obtenues sur les dynamiques des stades infestants sur le pâturage. Ces recommandations tiennent compte des situations spécifiques à chaque éleveur mais elles sont toutes fondées sur 4 grands principes : la durée de séjour sur une parcelle doit être inférieure à 7 jours, l'âge repousse doit être supérieur à 28 jours, l'irrigation des parcelles, quand elle est choisie, doit être réalisée 2 jours minimum après la sortie des animaux, la biomasse fourragère doit être maîtrisée par des charges suffisantes et des pratiques culturales.

Toutefois, il apparaît que la gestion rigoureuse du pâturage en tenant compte des impératifs pathologiques est une méthode complexe qui requiert une très grande technicité de la part de l'éleveur. En outre, il a été montré par simulation qu'en pâturage tournant, une erreur, même limitée, dans la rotation induisait une augmentation du risque d'infestation parasitaire en raison des variations très rapides dans le temps de la taille des populations de stades infestants.

Dans l'ensemble de la zone Caraïbe, la lutte chimique reste encore le moyen le plus utilisé. Mais l'emploi non raisonné et intensif a induit l'apparition très fréquente de résistances aux anthelminthiques pour le genre Haemonchus. Ces résistances d'Haemonchus contortus utkalensis ont été notées chez des ovins en Martinique (Gruner 1985, Gruner et al 1986, Bastien et al 1991) au Fenbendazole, au Febentel et à l'Oxfendazole dans $60 \%$ des élevages contrôlés. Une résistance du genre Haemonchus à l'ivermectine a été notée en 1995 en élevage intensif ovin en Martinique et caprin en Guadeloupe (G. Aumont et al, résultats non publiés). En Guadeloupe, 15 élevages caprins sur 20 contrôlés présentent des résistances des genres Haemonchus et Trichostrongylus au Fenbendazole (Amouroux 1994, N. Barré 1995, résultats non publiés). Une résistance du genre Haemonchus au Nétobimin (Hapadex, ND) a été observée en élevage intensif de caprins en Guadeloupe.

Le constat, d'une part de la très grande fréquence et de l'influence des strongyloses gastro-intestinales chez les caprins et les ovins dans les Antilles françaises quel que soit le mode d'élevage, d'autre part de l'inefficacité croissante de la lutte chimique, et enfin de la très grande difficulté de transmettre aux éleveurs des méthodes de gestion du pâturage rigoureuses et adaptées ont conduit à rechercher des moyens de lutte différents. Ainsi, en Martinique, l'association de bovins et d'ovins en croissance en pâturage intensif sur prairies irriguées diminue l'infestation par Haemonchus contortus, en particulier chez les agneaux mâles, permettant de diminuer par moitié la fréquence des traitements tout en augmentant la croissance et la production animale par unité de surface (Aumont et al 1995b, Mahieu et al 1995).

Un déterminisme génétique de la résistance des caprins Créoles aux strongles gastrointestinaux, principalement Haemonchus, a pu être mis en évidence dans des milieux irrigués ou secs (Mandonnet 1995, Mandonnet et al 1996). Ce résultat original, qui confirme des études menées en Afrique sur d'autres populations caprines (Baker 1997), a contribué à la poursuite d'études pour estimer des paramètres génétiques de cette résistance et quantifier l'intérêt de ce critère dans un schéma d'amélioration génétique. Enfin, un programme de recherche a été mis en place pour étudier les interrelations entre nutrition et strongyloses gastro-intestinales. Ces recherches devraient aboutir à des complémentations adaptées aux fourrages tropicaux permettant de renforcer la résistance et/ou la résilience (aptitude à la production malgré une infestation parasitaire) aux strongles gastro-intestinaux.

Les objectifs finalisés de ces recherches sont l'élaboration de plans de contrôle intégrés du parasitisme gastro-intestinal par la combinaison de traitements anthelminthiques, de techniques d'élevage, de gestion du pâturage et d'alimentation, et la sélection d'animaux plus résistants. Ces études représentent un enjeu déterminant pour l'intensification de l'élevage des petits ruminants, élément clef pour le maintien de cet élevage comme activité économiquement viable dans la zone Caraïbe. En effet, la maîtrise intégrée du parasitisme digestif chez ces espèces est une condition essentielle pour la réussite d'une intensification raisonnée des systèmes de pâturage en milieu tropical humide.

\section{Références bibliographiques}

Aberejola O.O., Schillhorn van Veen T.W., Njoku C.O., 1979. Ovine and caprine diseases in Nigeria : a review of economic losses. Bull. Anim. Hlth. Prod. Afr., 27, 65-75.

Alexandre G., Borel H., 1990. Les composantes de la production des petits ruminants aux Antilles. 3. Suivi de la conduite de l'alimentation au sein des exploitations caprines en Guadeloupe. Résultats et problèmes. Bull. Agron. Antilles-Guyane, 10, 17-25.

Alexandre G., Borel H., Matheron G., Rémy C., 1991. Elevages caprins en Guadeloupe. Rev. Elev. Méd. Vét. Pays Trop., (numéro spécial), 27-39.
Alexandre G., Aumont G., Fleury J., Mainaud J.C., Kandassamy T., 1997. Performances zootechniques de la chèvre Créole allaitante de Guadeloupe. Bilan de 20 ans dans un élevage expérimental de l'INRA. INRA Prod. Anim., 10, 7-20.

Amouroux I., 1994. Résistance des strongles gastrointestinaux aux anthelminthiques. Etude sur des caprins en Guadeloupe. Mémoire de fin d'études ISTOM, $61 \mathrm{p}$.

Aumont G., Gruner L., 1989. Population evolution of the free-living stage of goat gastrointestinal nematodes on herbage under tropical conditions in Guadeloupe (French West Indies). Int. J. Parasitol., 19, 539-546.
D'autres voies sont actuellement explorées : le pâturage en association avec des bovins, des complémentations alimentaires et l'amélioration génétique de la résistance des animaux aux strongyloses. 
Aumont G., Coulaud G., Grudé A., Gruner L., 1989. Pasture populations of cattle nematode larvae in Guadeloupe (French West Indies). Int. J. Parasitol., $19,547-554$

Aumont G., Gruner L., Berbigier P., 1991. Dynamique des populations de larves infestantes des strongles gastro-intestinaux des petits ruminants en milieu tropical humide. Conséquences sur la gestion des pâturages. Rev. Elev. Méd. Vét. Pays Trop., (numéro spécial), 123-131.

Aumont G., Barré N., Diaw S., Ouattara L., Pouillot R., Tillard E., Vassiliades G., 1995a. Preservation of fecal samples and laboratory sources of variation in fecal strongyles egg counts of small ruminants from tropical regions. In : Proc. of International Conference on Novel Approaches to the Control of Helminth Parasites of Livestock, Armidale, Australia 18-21 April 1995.

Aumont G., Mahieu M., Kojfer L., Pouillot R., Barré N., 1995b. Effects of grazing with heifers on gastrointestinal strongyles of sheep in irrigated Digitaria decumbens pastures in Martinique (F.W.I.). In : Proc. of International Conference on Novel Approaches to the Control of Helminth Parasites of Livestock, Armidale, Australia 18-21 April 1995.

Aumont G., Frauli D., Simon R., Pouillot R., Diaw S., Mandonnet N., 1996a. Comparisons of methods for counting infective third stage larvae of gastrointestinal nematodes of small ruminants in pastures under tropical conditions. Vet. Parasitol., 62, 307-315.

Aumont G., Pouillot R., Simon R., Barré N., 1996b. Epidemiology of digestive parasitism of goat in farm of Guadeloupe (F.W.I.). In : Proceedings of the VIth International Conference on Goat, Beijing, 5-11 may 1996, China, Intern. Acad. Publ. ed., 738-741.

Baker L., 1997. Résistance génétique des petits ruminants aux helminthes en Afrique. INRA Prod. Anim., 10, 99-110.

Banks D.J.R., Singh R., Barger I.A., Pratap B., LeJambre L.F., 1990. Development and survival of infective larvae of Haemonchus contortus and Trichostrongylus colubriformis on pasture in a tropical environment. Int. J. Parasitol., 20, 155-160.

Bastien O., Kerbœuf D., Leimbacher F., Gevrey J., Nicolas J.A., Hubert J., Heinrich O., 1991. Recherche des causes d'échecs thérapeutiques de la lutte contre les strongyloses gastro-intestinales des ovins en Martinique. Rev. Elev. Méd. Vét. Pays Trop., (numéro spécial), 117-122.

Berbigier P., Gruner L., Mambrini M., Sophie S.A., 1990. Faecal water content and egg survival of goat gastro-intestinal strongyles under dry tropical conditions in Guadeloupe. Parasitol. Res., 76, 379-385.

Camus E., 1991. La cowdriose caprine et ovine en Guadeloupe. Rev. Elev. Méd. Vét. Pays Trop., (numéro spécial), 139-143.

Chemineau P., Cognié Y., Xandé A., Peroux F., Alexandre G., Levy F., Shitalou E., Beche J.M., Sergent D., Camus E., Barré N., Thimonier J., 1984. Le « cabrit Créole » de Guadeloupe et ses caractéristiques zootechniques : monographie. Rev. Elev. Méd. Vét. Pays Trop., 37, 225-238.

Courtney C.H., Wildeus S., 1991. Seasonal transmission of gastro-intestinal nematodes of sheep on St Croix. In : Proc. Symp. Hair Sheep Res., 28-29 June 1991, University of the Virgin Islands ed., 357-362.

Das R.W., Witlock J.H., 1960. Subspeciation in Haemonchus contortus (Rudolphi, 1803) Nematoda Trichostrongylidea. Corn. Vet., 50, 182-197.
Durand P., Lenoir J.M., Liabœuf J.M., Leimbacher F., 1983. Dépistage des problèmes d'importance économique chez les petits ruminants des Antilles françaises, Rapport COPELCOG, Guadeloupe, 27 p.

Esterre P., Maitre J.M., 1985. Les affections parasitaires des ruminants en Guadeloupe. Rev. Elev. Méd. Vét. Pays Trop., 38, 49-53.

Euzeby J., Graber M., 1973. Enquête parasitologique en Guadeloupe. Bull. Soc. Path. Exo., 66, 558-567.

Fabiyi J.P., 1987. Production losses and control of helminths in ruminants of tropical regions. Int. J. Parasitol., 17, 435-442.

Faugère O., Tillard E., Faugère B., Merlin P., 1991. Prophylaxies chez les petits ruminants au Sénégal : évaluation technico-économique de leurs effets en milieu villageois. Document de travail P.P.R., $\mathrm{n}^{\circ} 34$.

Gruner L., 1985. Contrôle des strongyloses digestives des petits ruminants aux Antilles françaises : développement de résistances aux benzimidazoles et intérêt d'une gestion raisonnée des pâturages. Rev. Elev. Méd. Vét. Pays Trop., 38, 386-393.

Gruner L., Peroux F., Aumont G., 1984a. Dynamique des populations de parasites internes dans un élevage semi-intensif de chèvres Créoles en Guadeloupe. In : Les maladies de la chèvre, 695-704. INRA, Paris.

Gruner L., Peroux F., Chemineau P., 1984b. Distribution et rôle de l'Haemonchose dans un élevage semi-intensif de chevreaux de race Créole en Guadeloupe. In : Les maladies de la chèvre, 705-715. INRA, Paris.

Gruner L., Kerbœuf D., Beaumont C., Hubert J., 1986. Resistance to benzimidazole of Haemonchus contortus utkalensis on Martinique. Vet. Rec., 118, 276.

Gruner L., Berbigier P., Cortet J., Sauve C., 1989. Effects of irrigation on appearance and survival of infective larvae of goat gastro-intestinal nematodes in Guadeloupe (French West indies). Int. J. Parasitol., 19, 409-415.

Ikeme M.M., Fatimah I., Lee C.C., 1986. Seasonal incidence of infective nematode larvae on pasture and nematode worm population of goats in Selangor. Kajian Vet., 18, 69-76.

INSEE, 1993. Tableaux Economiques Régionaux édition 1993, Pointe-à-Pitre, I.N.S.E.E. AntillesGuyane, 136 p.

Krecek R.C., Grœneveld H.T., van Wik J.A., 1991. Effects of time of day, season and stratum on Haemonchus contortus and Haemonchus placei third stage larvae on irrigated pasture. Vet. Parasitol., 40, 87-98.

Lasserre G., 1961. La Guadeloupe, tome I : Le milieu naturel et l'histoire ; tome II : Les îles et leurs problèmes. Bordeaux, Thèse Doctorat Géographie, Université de Bordeaux, $121 \mathrm{p}$.

Lasserre G., 1982. Atlas des départements français d'Outre mer : La Guadeloupe. 36 planches. Editions du CNRS, Paris.

Lebigre C., 1979. L'élevage caprin à la Guadeloupe. Thèse Doct. vét., ENV Maisons-Alfort, 179 p.

Leimbacher F., Tatareau J.C., 1991. Evolution des résultats de contrôle de performance des ovins à la Martinique. Rev. Elev. Méd. Vét. Pays Trop., (numéro spécial), 69-74.

Levine N.D., Todd K.S., 1975. Micrometeorological factors involved in development and survival of freeliving stages of the sheep nematodes Haemonchus contortus and Trichostrongylus colubriformis on pasture. Int. J. Biometeor., 19, 174-183. 
Levine N.D., Todd K.S., Boatman P.A., 1977. Development and survival of Haemonchus contortus on pasture. Am. J. Vet. Res., 35, 1413-1422.

Mahieu M., 1991. Impact of irrigation on performances of Martinique hair sheep grazing pastures of Digitaria decumbens (Stent.) in southeastern Martinique. In : Proc. Symp. Hair Sheep Res., 28-29 June 1991, University of the Virgin Island ed., 101-108.

Mahieu M., Aumont G., Alexandre G., Fesneau X., Boval M., Mandonne M., Kojfer L., 1995. Mixed grazing by sheep and steers of irrigated Digitaria decumbens pastures in Martinique (F.W.I.). Ann. Zootech., 44 (Suppl), 343.

Mahieu M., Aumont G., Alexandre G., 1997. Elevage intensif des ovins tropicaux à la Martinique. INRA Prod. Anim., 10, 21-32.

Mandonnet N., 1995. Analyse de la variabilité génétique de la résistance aux strongles gastro-intestinaux chez les petits ruminants. Eléments pour la définition d'objectifs et de critères de sélection en milieu tempéré ou tropical. Thèse Doct. ès-sciences, Université Paris Sud, Orsay, 108 p.

Mandonnet N., Aumont G., Fleury J., Gruner L., Bouix J., Vu Tien Khang J., 1996. Genetic variability in resistance of creole goat to natural infection with Trichostrongylids in Guadeloupe. Ann. New York Acad. Sci., 791, 421-431.

Messean A., 1981. Modélisation du cycle d'évolution de nématodes parasites d'ovins. In : Biométrie et Epidémiologie, Legay \& Tommassone eds., 28-43. INRA, Paris.

Pépin L., 1994. Recherche de polymorphisme génétique chez les caprins. Applications à l'étude de la diversité des populations, au contrôle de la filiation et à la résistance génétique à la cowdriose. Thèse Doct. ès-sciences, Univ. Paris Sud, Orsay, 139 p.

Peroux F., 1982. Epidémiologie des parasitoses gastro-intestinales des caprins à la Guadeloupe. Thèse Doct. vét., ENV Maisons-Alfort, 75 p.

Petitclerc M., Lefevre P.C., Calvez D., Couderc P., Liabœuf J.M., Camus E., 1991. Quelques aspects de la pathologie des petits ruminants en Guadeloupe et en Martinique. Rev. Elev. Méd. Vét. Pays Trop., (numéro spécial), 113-115.

Pouillot R., 1995. Epidémiologie des strongyloses gastro-intestinales des caprins Créoles en Guadeloupe, saison humide. Thèse Doct. vét., ENV Maisons-Alfort, $100 \mathrm{p}$.

Schillhorn Van Veen T.W., 1973. Small ruminants health problems in Northern Nigeria with emphasis on helminthiasis. Nigerian Vet. J., 2, 26-31.

Simon R., 1994. Epidémiologie des strongyloses gastro-intestinales des caprins Créoles en Guadeloupe. DEA Parasitologie, $25 \mathrm{p}$.

Simon R., Aumont G., Aprelon R., 1996. Variations in herbage density of third stage larvae of goats strongyle during dry season in Guadeloupe (F.W.I.). Ann. New York Acad. Sci., 791, 412-420.

Singh S., 1993. Endoparasites of sheep and goats in Trinidad. B. Sc. thesis, Agriculture Degree, University of the West Indies, $52 \mathrm{p}$.

Tatareau J.C., Laleau G., Pensedent-Erblon J., Shitalou E., Milhet P., Barré N., Matheron G., 1991. L'élevage des petits ruminants en Martinique, Guadeloupe et Guyane : situation actuelle. Rev. Elev. Méd. Vét. Pays Trop., (numéro spécial), 5-10.

Vassiliades G., 1984. Le parasitisme gastro-intestinal chez le mouton du Sénégal. Rev. Elev. Méd. Vét. Pays Trop., 34, 169-177.

Waller P.J., Donald A.D., 1970. The response to dessication of eggs Trichostrongylus colubriformis (Nematoda, Trichostrongylidae). Parasitology, 61, 195-204.

Wharton D.A., 1982. The survival of dessication by the free-living stages Trichostrongylus colubriformis (Nematoda, Trichostrongylidae). Parasitology, 84, 455-402.

Williams H., 1990. Diseases of ruminants in the caribbean with special focus on ticks and tick associated diseases. In : Cowdriosis and dermatophilosis of livestock in the caribbean region, Antigua, C.T.A. seminar proceeding, 12-14 Nov. 1990, CARDI and CTA eds, 31-62.

\section{Abstract}

Digestive parasitism of small ruminants in the French West Indies.

The Nematode Haemonchus contortus, the Cestode Moniezia sp. and the protozoa Eimeria spp. are the main digestive parasites of small ruminants in the Caribbean. Models of infective third stage larvae (L3) of Haemonchus contortus and Trichostrongylus colubriformis have been established to use simulation to estimate the infestation risk according to season, irrigation practice, herbage mass and pasture management.

The faunistic diversity is low : 10 species of worm in Guadeloupe. The prevalence of Haemonchus sp. and Trichostrongylus spp. range from 80 to $100 \%$. Incidences of these worms are higher than $60 \%$ per month. The main sources of variation in infestation risk are irrigation, climate and susceptibility of animals. The strongylosis is responsible for more than $80 \%$ of the mortality rate before weaning, which raises to $40 \%$. Resistance of Haemon- chus contortus strains to anthelmintics (benzimidazoles) are common in Guadeloupe and Martinique. All the studies on farms confirmed that digestive strongylosis is the main disease of small ruminants in the Lesser Antilles.

The research carried out by the Animal Production Research Unit of INRA to improve animal breeding and husbandry in pasture (type of forage, stocking rate recommendations, mixed grazing, etc...) took into account the importance of strongylosis to small ruminants production. The genetic resistance of creole goats to digestive strongles and the possible use of feeding supplements are being studied for integration in a plan to control these parasitic diseases.

AUMONT G., POUILLOT R., SIMON R., HOSTACHE G., VARO H., BARRÉ N., 1997. Parasitisme digestif des petits ruminants dans les Antilles françaises. INRA Prod. Anim., 10 (1), 79-89. 